\title{
Azimuthal Anisotropy and Correlations in the Hard Scattering Regime at RHIC
}

C. Adler ${ }^{11}$, Z. Ahammed ${ }^{23}$, C. Allgower ${ }^{12}$, J. Amonett ${ }^{14}$, B.D. Anderson ${ }^{14}$, M. Anderson ${ }^{5}$, G.S. Averichev ${ }^{9}$, J. Balewski ${ }^{12}$, O. Barannikova ${ }^{9,23}$, L.S. Barnby ${ }^{14}$, J. Baudot ${ }^{13}$, S. Bekele ${ }^{20}$, V.V. Belaga ${ }^{9}$, R. Bellwied ${ }^{31}$, J. Berger ${ }^{11}$, H. Bichsel ${ }^{30}$, A. Billmeier ${ }^{31}$, L.C. Bland ${ }^{2}$, C.O. Blyth ${ }^{3}$, B.E. Bonner ${ }^{24}$, A. Boucham ${ }^{26}$, A. Brandin ${ }^{18}$, A. Bravar ${ }^{2}$, R.V. Cadman ${ }^{1}$, H. Caines ${ }^{33}$, M. Calderón de la Barca Sánchez ${ }^{2}$, A. Cardenas ${ }^{23}$, J. Carroll ${ }^{15}$, J. Castillo ${ }^{26}$, M. Castro ${ }^{31}$, D. Cebra ${ }^{5}$, P. Chaloupka ${ }^{20}$, S. Chattopadhyay ${ }^{31}$, Y. Chen ${ }^{6}$, S.P. Chernenko ${ }^{9}$, M. Cherney ${ }^{8}$, A. Chikanian ${ }^{33}$, B. Choi $^{28}$, W. Christie ${ }^{2}$, J.P. Coffin ${ }^{13}$, T.M. Cormier ${ }^{31}$, J.G. Cramer ${ }^{30}$, H.J. Crawford ${ }^{4}$, W.S. Deng ${ }^{2}$, A.A. Derevschikov ${ }^{22}$, L. Didenko ${ }^{2}$, T. Dietel ${ }^{11}$, J.E. Draper ${ }^{5}$, V.B. Dunin ${ }^{9}$, J.C. Dunlop ${ }^{33}$, V. Eckardt ${ }^{16}$, L.G. Efimov ${ }^{9}$, V. Emelianov ${ }^{18}$, J. Engelage ${ }^{4}$, G. Eppley ${ }^{24}$, B. Erazmus ${ }^{26}$, P. Fachini ${ }^{2}$, V. Faine ${ }^{2}$, J. Faivre ${ }^{13}$, K. Filimonov ${ }^{15}$, E. Finch ${ }^{33}$, Y. Fisyak ${ }^{2}$, D. Flierl ${ }^{11}$, K.J. Foley ${ }^{2}$, J. Fu ${ }^{15,32}$, C.A. Gagliardi ${ }^{27}$, N. Gagunashvili ${ }^{9}$, J. Gans ${ }^{33}$, L. Gaudichet ${ }^{26}$, M. Germain ${ }^{13}$, F. Geurts ${ }^{24}$, V. Ghazikhanian ${ }^{6}$, O. Grachov ${ }^{31}$, V. Grigoriev ${ }^{18}$, M. Guedon ${ }^{13}$, E. Gushin ${ }^{18}$, T.J. Hallman ${ }^{2}$, D. Hardtke ${ }^{15}$, J.W. Harris ${ }^{33}$, T.W. Henry ${ }^{27}$, S. Heppelmann ${ }^{21}$,

T. Herston ${ }^{23}$, B. Hippolyte ${ }^{13}$, A. Hirsch ${ }^{23}$, E. Hjort ${ }^{15}$, G.W. Hoffmann ${ }^{28}$, M. Horsley ${ }^{33}$, H.Z. Huang 6 , T.J. Humanic ${ }^{20}$, G. Igo ${ }^{6}$, A. Ishihara ${ }^{28}$, Yu.I. Ivanshin ${ }^{10}$, P. Jacobs ${ }^{15}$, W.W. Jacobs ${ }^{12}$, M. Janik ${ }^{29}$, I. Johnson ${ }^{15}$, P.G. Jones ${ }^{3}$, E.G. Judd ${ }^{4}$, M. Kaneta ${ }^{15}$, M. Kaplan ${ }^{7}$, D. Keane ${ }^{14}$, J. Kiryluk ${ }^{6}$, A. Kisiel ${ }^{29}$, J. Klay ${ }^{15}$, S.R. Klein ${ }^{15}$, A. Klyachko ${ }^{12}$, A.S. Konstantinov ${ }^{22}$, M. Kopytine ${ }^{14}$, L. Kotchenda ${ }^{18}$, A.D. Kovalenko ${ }^{9}$, M. Kramer ${ }^{19}$, P. Kravtsov $^{18}$, K. Krueger ${ }^{1}$, C. Kuhn ${ }^{13}$, A.I. Kulikov ${ }^{9}$, G.J. Kunde ${ }^{33}$, C.L. Kunz ${ }^{7}$, R.Kh. Kutuev ${ }^{10}$, A.A. Kuznetsov ${ }^{9}$, L. Lakehal-Ayat ${ }^{26}$, M.A.C. Lamont ${ }^{3}$, J.M. Landgraf ${ }^{2}$, S. Lange ${ }^{11}$, C.P. Lansdell ${ }^{28}$, B. Lasiuk ${ }^{33}$, F. Laue ${ }^{2}$, J. Lauret ${ }^{2}$, A. Lebedev ${ }^{2}$, R. Lednický ${ }^{9}$, V.M. Leontiev ${ }^{22}$, M.J. LeVine ${ }^{2}$, Q. Li ${ }^{31}$, S.J. Lindenbaum ${ }^{19}$, M.A. Lisa ${ }^{20}$,

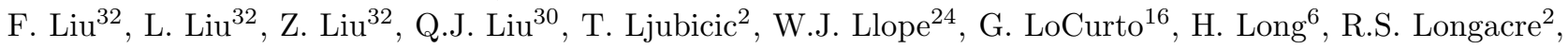
M. Lopez-Noriega ${ }^{20}$, W.A. Love ${ }^{2}$, T. Ludlam² ${ }^{2}$ D. Lynn $^{2}$, J. Ma ${ }^{6}$, R. Majka ${ }^{33}$, S. Margetis ${ }^{14}$, C. Markert ${ }^{33}$, L. $\operatorname{Martin}^{26}$, J. Marx ${ }^{15}$, H.S. Matis ${ }^{15}$, Yu.A. Matulenko ${ }^{22}$, T.S. McShane ${ }^{8}$, F. Meissner ${ }^{15}$, Yu. Melnick ${ }^{22}$, A. Meschanin ${ }^{22}$, M. Messer ${ }^{2}$, M.L. Miller ${ }^{33}$, Z. Milosevich ${ }^{7}$, N.G. Minaev ${ }^{22}$, J. Mitchell24, V.A. Moiseenko ${ }^{10}$, C.F. Moore ${ }^{28}$, V. Morozov ${ }^{15}$, M.M. de Moura ${ }^{31}$, M.G. Munhoz ${ }^{25}$, J.M. Nelson ${ }^{3}$, P. Nevski ${ }^{2}$, V.A. Nikitin ${ }^{10}$, L.V. Nogach $^{22}$, B. Norman ${ }^{14}$, S.B. Nurushev ${ }^{22}$, G. Odyniec ${ }^{15}$, A. Ogawa ${ }^{21}$, V. Okorokov ${ }^{18}$, M. Oldenburg ${ }^{16}$, D. Olson ${ }^{15}$, G. Paic ${ }^{20}$, S.U. Pandey ${ }^{31}$, Y. Panebratsev ${ }^{9}$, S.Y. Panitkin ${ }^{2}$, A.I. Pavlinov ${ }^{31}$, T. Pawlak ${ }^{29}$, V. Perevoztchikov ${ }^{2}$, W. Peryt ${ }^{29}$, V.A Petrov ${ }^{10}$, M. Planinic ${ }^{12}$, J. Pluta ${ }^{29}$, N. Porile ${ }^{23}$, J. Porter ${ }^{2}$, A.M. Poskanzer ${ }^{15}$, E. Potrebenikova ${ }^{9}$, D. Prindle ${ }^{30}$, C. Pruneau ${ }^{31}$, J. Putschke ${ }^{16}$, G. Rai ${ }^{15}$, G. Rakness ${ }^{12}$, O. Ravel ${ }^{26}$, R.L. Ray ${ }^{28}$, S.V. Razin ${ }^{9,12}$, D. Reichhold ${ }^{8}$, J.G. Reid ${ }^{30}$, G. Renault ${ }^{26}$, F. Retiere ${ }^{15}$, A. Ridiger ${ }^{18}$, H.G. Ritter ${ }^{15}$, J.B. Roberts ${ }^{24}$,

O.V. Rogachevski ${ }^{9}$, J.L. Romero ${ }^{5}$, A. $R^{2}{ }^{31}$, C. Roy $^{26}$, V. Rykov ${ }^{31}$, I. Sakrejda ${ }^{15}$, S. Salur ${ }^{33}$, J. Sandweiss ${ }^{33}$, A.C. Saulys ${ }^{2}$, I. Savin ${ }^{10}$, J. Schambach ${ }^{28}$, R.P. Scharenberg ${ }^{23}$, N. Schmitz ${ }^{16}$, L.S. Schroeder ${ }^{15}$, A. Schüttauf ${ }^{16}$,

K. Schweda ${ }^{15}$, J. Seger ${ }^{8}$, D. Seliverstov ${ }^{18}$, P. Seyboth ${ }^{16}$, E. Shahaliev ${ }^{9}$, K.E. Shestermanov ${ }^{22}$, S.S. Shimanskii ${ }^{9}$, V.S. Shvetcov ${ }^{10}$, G. Skoro ${ }^{9}$, N. Smirnov ${ }^{33}$, R. Snellings ${ }^{15}$, P. Sorensen ${ }^{6}$, J. Sowinski ${ }^{12}$, H.M. Spinka ${ }^{1}$, B. Srivastava ${ }^{23}$, E.J. Stephenson ${ }^{12}$, R. Stock ${ }^{11}$, A. Stolpovsky ${ }^{31}$, M. Strikhanov ${ }^{18}$, B. Stringfellow ${ }^{23}$, C. Struck ${ }^{11}$, A.A.P. Suaide ${ }^{31}$, E. Sugarbaker $^{20}$, C. Suire ${ }^{2}$, M. Sumbera ${ }^{20}$, B. Surrow ${ }^{2}$, T.J.M. Symons ${ }^{15}$, A. Szanto de Toledo ${ }^{25}$, P. Szarwas ${ }^{29}$, A. Tai ${ }^{6}$,

J. Takahashi ${ }^{25}$, A.H. Tang ${ }^{14}$, J.H. Thomas ${ }^{15}$, M. Thompson ${ }^{3}$, V. Tikhomirov ${ }^{18}$, M. Tokarev ${ }^{9}$, M.B. Tonjes ${ }^{17}$,

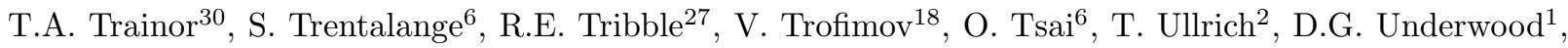

G. Van Buren ${ }^{2}$, A.M. VanderMolen ${ }^{17}$, I.M. Vasilevski ${ }^{10}$, A.N. Vasiliev ${ }^{22}$, S.E. Vigdor ${ }^{12}$, S.A. Voloshin ${ }^{31}$,

F. Wang ${ }^{23}$, H. Ward ${ }^{28}$, J.W. Watson ${ }^{14}$, R. Wells ${ }^{20}$, G.D. Westfall ${ }^{17}$, C. Whitten Jr. ${ }^{6}$, H. Wieman ${ }^{15}$, R. Willson ${ }^{20}$, S.W. Wissink ${ }^{12}$, R. Witt ${ }^{33}$, J. Wood ${ }^{6}$, N. Xu ${ }^{15}$, Z. Xu ${ }^{2}$, A.E. Yakutin ${ }^{22}$, E. Yamamoto ${ }^{15}$, J. Yang ${ }^{6}$, P. Yepes ${ }^{24}$, V.I. Yurevich ${ }^{9}$, Y.V. Zanevski ${ }^{9}$, I. Zborovský9 ${ }^{9}$, H. Zhang ${ }^{33}$, W.M. Zhang ${ }^{14}$, R. Zoulkarneev ${ }^{10}$, A.N. Zubarev ${ }^{9}$

(STAR Collaboration)

\footnotetext{
${ }^{1}$ Argonne National Laboratory, Argonne, Illinois 60439

${ }^{2}$ Brookhaven National Laboratory, Upton, New York 11973

${ }^{3}$ University of Birmingham, Birmingham, United Kingdom

${ }^{4}$ University of California, Berkeley, California 94720

${ }^{5}$ University of California, Davis, California 95616

${ }^{6}$ University of California, Los Angeles, California 90095

${ }^{7}$ Carnegie Mellon University, Pittsburgh, Pennsylvania 15213

${ }^{8}$ Creighton University, Omaha, Nebraska 68178

${ }^{9}$ Laboratory for High Energy (JINR), Dubna, Russia

${ }^{10}$ Particle Physics Laboratory (JINR), Dubna, Russia

${ }^{11}$ University of Frankfurt, Frankfurt, Germany
} 




(Dated: November 5, 2018)

\begin{abstract}
Azimuthal anisotropy $\left(v_{2}\right)$ and two-particle angular correlations of high $p_{T}$ charged hadrons have been measured in $\mathrm{Au}+\mathrm{Au}$ collisions at $\sqrt{s_{N N}}=130 \mathrm{GeV}$ for transverse momenta up to $6 \mathrm{GeV} / \mathrm{c}$, where hard processes are expected to contribute significantly. The two-particle angular correlations exhibit elliptic flow and a structure suggestive of fragmentation of high $p_{T}$ partons. The monotonic rise of $v_{2}\left(p_{T}\right)$ for $p_{T}<2 \mathrm{GeV} / \mathrm{c}$ is consistent with collective hydrodynamical flow calculations. At $p_{T}>3 \mathrm{GeV} / \mathrm{c}$ a saturation of $v_{2}$ is observed which persists up to $p_{T}=6 \mathrm{GeV} / \mathrm{c}$.
\end{abstract}

PACS numbers: 25.75.Ld

Collisions of heavy nuclei at ultra-relativistic energies exhibit strong collective flow effects indicative of a volume of hot matter so dense that descriptions involving hydrodynamic behavior in a locally thermalized system may apply [1]. The azimuthal anisotropy of final state hadrons in non-central collisions 2] is sensitive to the system evolution at early times [3]. At high $p_{T}$, a hydrodynamic description of the system may break down as processes involving hard scattering of the initial-state partons are expected to play the dominant role.

Calculations based on perturbative QCD predict that high energy partons traversing nuclear matter lose energy through induced gluon radiation [4], where the magnitude of the energy loss is dependent upon the density of the medium [5]. Recent measurements of inclusive charged hadron distributions in $\mathrm{Au}+\mathrm{Au}$ collisions at $\sqrt{s_{N N}}=130 \mathrm{GeV}$ show a suppression of hadron yields at high $p_{T}$ in central collisions relative to peripheral collisions and scaled nucleon-nucleon interactions, consistent with the picture of partonic energy loss in a dense system [6, 7]. The fragmentation products of partons that have propagated through the azimuthally asymmetric system generated by non-central collisions may exhibit azimuthal anisotropy due to energy loss and the azimuthal dependence of the path length, providing important information about the initial conditions and dynamics in a heavy ion collision $[8,9]$.
The azimuthal anisotropy of an event in momentum space is quantified by the coefficients of the Fourier decomposition of the azimuthal particle distribution with respect to the reaction plane, with the second harmonic coefficient $v_{2}$ referred to as elliptic flow. These coefficients can be inferred from the particle distribution with respect to the estimated reaction plane orientation, corrected for the reaction plane resolution, or from twoparticle correlation analysis [10]. The methods are identical if the azimuthal correlation between particles results solely from their correlation with the reaction plane. Correlations that are localized in both rapidity and azimuthal angle are characteristic of high energy partons fragmenting into jets of hadrons. Such short-range correlations may be isolated from elliptic flow using twoparticle correlation analyses performed in different regions of relative pseudorapidity.

The transverse momentum dependence of $v_{2}$ has been previously measured in $\mathrm{Au}+\mathrm{Au}$ collisions at $\sqrt{s_{N N}}=$ $130 \mathrm{GeV}$ for charged 1] and identified 11] particles in the region of $p_{T}<2 \mathrm{GeV} / \mathrm{c}$. Elliptic flow at RHIC can be described by a hydrodynamical model for $p_{T}$ up to $2 \mathrm{GeV} / \mathrm{c}$. In this Letter we report the first results on $v_{2}\left(p_{T}\right)$ of charged particles measured in this reaction up to $p_{T}=6 \mathrm{GeV} / \mathrm{c}$, together with the analysis of twoparticle azimuthal correlations among high $p_{T}$ charged particles. 
The Solenoidal Tracker at RHIC (STAR) consists of several detector subsystems in a large solenoidal magnet. The main tracking detector is the Time Projection Chamber (TPC), which has wide acceptance in pseudorapidity and complete azimuthal coverage [12]. For this analysis, the full data set from the first year data taking of the STAR experiment was used, consisting of $300 \mathrm{~K}$ minimum-bias and $400 \mathrm{~K}$ centrally triggered events. The minimum-bias data contain hadronic $\mathrm{Au}+\mathrm{Au}$ interactions at $\sqrt{s_{N N}}=130 \mathrm{GeV}$ corresponding to $\sim 90 \%$ of the geometric cross section $\sigma_{\text {geo }}$, while the centrally triggered data provide an unbiased event sample for the most central $10 \%$ of the minimum-bias data set. The trigger conditions and event and track selection cuts for $v_{2}$ analysis are identical to those used previously [11].

The reaction plane analysis method involves the calculation of the orientation of the event plane, which is an experimental estimator of the true reaction plane angle. For this analysis, the second harmonic event plane angles, $\Psi_{2}$, were calculated for the full event and two subevents, consisting of randomly selected particles from the same event. The results are insensitive to the selection method (random, pseudorapidity, or charge sign) for assigning particles to subevents [1].

Jets may bias the reconstruction of the reaction plane if the intra-jet correlations produce asymmetries which are of similar magnitude to that due to collective flow. Systematic studies were undertaken to assess the bias. In general, the products of jet fragmentation have higher $p_{T}$ than other particles produced in a collision [13]. Only low $p_{T}$ particles $\left(p_{T}<2 \mathrm{GeV} / \mathrm{c}\right)$ were selected to calculate the event plane orientation. Using $p_{T}$-cutoffs of $1.5,1.0$ and $0.5 \mathrm{GeV} / \mathrm{c}$ resulted in statistically consistent $v_{2}$ values. In addition, two sets of events were analyzed, the first containing only events with a particle of $p_{T}>3$ $\mathrm{GeV} / \mathrm{c}$ and the second with all events, separately. The values of $v_{2}$ as a function of $p_{T}$ were the same in both data sets. To ensure that particles produced within a jet do not affect the reaction plane reconstruction, all subevent particles in a pseudorapidity region of $|\Delta \eta|<0.5$ around the highest $p_{T}$ particle in the event were excluded and the results were insensitive to this procedure. Varying the track selection criteria (distance of closest approach to the primary vertex, number of measured space points, etc.) also made no significant difference.

Finite momentum resolution at high transverse momenta combined with a rapid decrease of hadron yield with increasing $p_{T}$ may cause flattening of the $p_{T^{-}}$ dependence of $v_{2}$. The momentum resolution has been determined by embedding simulated single tracks into real raw data events. For the cuts used in this analysis, the momentum resolution for $p_{T}>1.5 \mathrm{GeV} / \mathrm{c}$ is parameterized as $\delta p_{T} / p_{T}=0.013+0.014 p_{T} /(\mathrm{GeV} / \mathrm{c})$ for central events at magnetic field $B=0.25 \mathrm{~T}$. We have studied the possible effects of the momentum resolution on $v_{2}$ at high transverse momenta using a Monte-Carlo simulation by generating particles with a power-law $p_{T}$-distribution [7] and with various $v_{2}\left(p_{T}\right)$ dependencies. The estimated relative systematic error on $v_{2}$ due to momentum resolution is $5 \%$ at $p_{T}=5 \mathrm{GeV} / \mathrm{c}$.

The reaction plane analysis integrates all possible sources of azimuthal correlations, including those unrelated to the orientation of the reaction plane. The non-flow correlations may be due to resonance decays, (mini)jets, final state interactions (particularly Coulomb effects), momentum conservation, etc. The strength of non-flow contributions relative to the measured azimuthal asymmetry was discussed in [1], which estimated that $15-20 \%$ of the $v_{2}$ signal obtained with a reaction plane analysis method is due to non-flow correlations. A four-particle correlation method [14] for flow measurements reduces non-flow sources to a negligible level. The centrality averaged values of $v_{2}$ from four-particle correlations are $15 \%$ lower than those obtained from the reaction plane analysis 15. The ratio of $v_{2}$ from the two methods is approximately independent of pseudorapidity and transverse momentum within $0.1<p_{T}<4.0$ $\mathrm{GeV} / \mathrm{c}$, the range accessible to the four-particle correlation method with the current statistics [15]. Based on these studies, we assign a ${ }_{-20}^{+5} \%$ systematic uncertainty to the $v_{2}$ values presented. This uncertainty is highly correlated point-to-point at all $p_{T}$-values.

Figure 11 shows the azimuthal distributions with respect to the reaction plane of charged particles within $2<p_{T}<6 \mathrm{GeV} / \mathrm{c}$, for three collision centralities. The distributions are corrected for the reaction plane resolution. All distributions exhibit the second harmonic behavior characteristic of elliptic flow. A fit of distributions by $1+2 v_{2} \cos 2\left(\phi_{\text {lab }}-\Psi_{\text {plane }}\right)$ yields $v_{2}=$ $0.218 \pm 0.003,0.162 \pm 0.002,0.090 \pm 0.001$ for the three centrality bins. The errors are statistical only. There is large azimuthal anisotropy at high $p_{T}$ for all centralities.

Figure 2 shows the differential elliptic flow $v_{2}$ as a

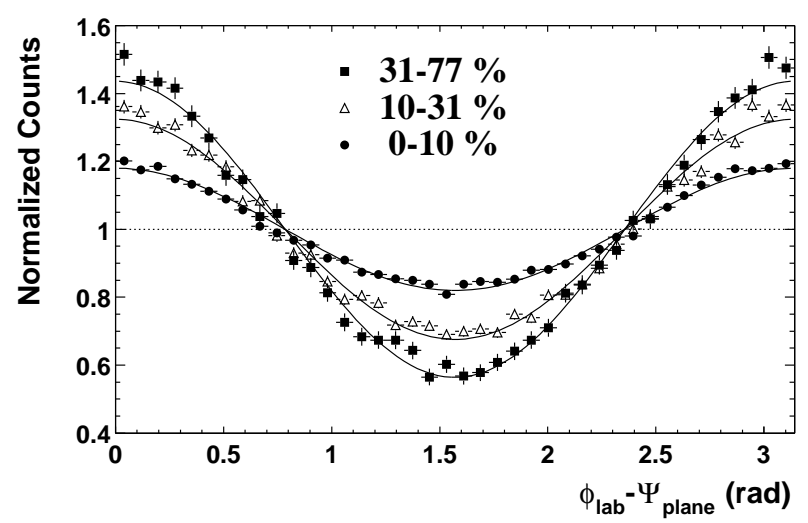

FIG. 1: Azimuthal distributions with respect to the reaction plane of charged particles within $2<p_{T}<6 \mathrm{GeV} / \mathrm{c}$, for three collision centralities. The percentages are given with respect to the geometrical cross section $\sigma_{\text {geo }}$. Solid lines show fits by $1+2 v_{2} \cos 2\left(\phi_{\text {lab }}-\Psi_{\text {plane }}\right)$. 


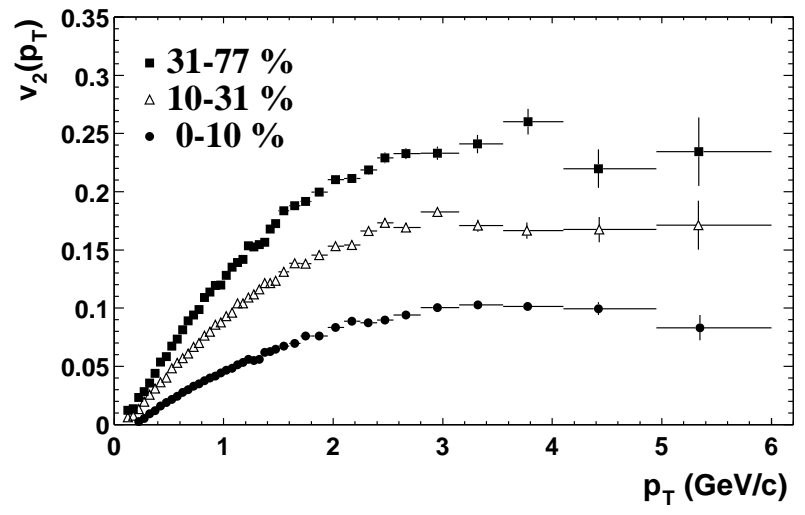

FIG. 2: $v_{2}\left(p_{T}\right)$ for different collision centralities. The errors are statistical only. The systematic uncertainties, which are highly correlated point-to-point, are ${ }_{-20}^{+5} \%$.

function of $p_{T}$ for three collision centralities. The values of $v_{2}\left(p_{T}\right)$ were obtained from the second moment of the distribution of particles with respect to the reaction plane, i.e. from the average values $\left\langle\cos 2\left(\phi_{l a b}-\Psi_{\text {plane }}\right)\right\rangle$, corrected for the reaction plane resolution. At a given $p_{T}$, the more peripheral collisions have larger $v_{2}$. For all centralities, $v_{2}$ rises linearly up to $p_{T}=1 \mathrm{GeV} / \mathrm{c}$, then deviates from a linear rise and saturates for $p_{T}>3$ $\mathrm{GeV} / \mathrm{c}$. The saturation persists up to $6 \mathrm{GeV} / \mathrm{c}$ and is in contrast to non-dissipative hydrodynamical calculations, which predict a continuous rise of $v_{2}$ with increasing transverse momentum [16].

In Ref. 9], the particle production is decomposed into phenomenological "soft" and perturbative QCD calculable hard components. The soft nonperturbative component incorporates hydrodynamic elliptic flow, whereas the pQCD calculable part includes energy loss (jet quenching). In this model the magnitude of $v_{2}$ at high $p_{T}$ is sensitive to nuclear geometry and the initial gluon density achieved in a collision. Figure 3] compares the minimum-bias differential elliptic flow $v_{2}\left(p_{T}\right)$ with calculations from Ref. 9]. These calculations also predict a decrease of $v_{2}$ with increasing $p_{T}$ at high transverse momenta. This decrease should even be stronger if transverse expansion of the system is taken into account [17]. A rapid expansion dilutes the initial coordinate space azimuthal asymmetry resulting in a reduction of the measured azimuthal anisotropies due to energy loss. A flavor dependence of both the asymmetry and $p_{T}$ differential particle multiplicities has been suggested as one of the possible scenarios for the $v_{2}\left(p_{T}\right)$ behavior [17]. The observed saturation of $v_{2}$ at $p_{T} \sim 2-3 \mathrm{GeV} / \mathrm{c}$ can be quantitatively reproduced in a parton cascade model with only elastic rescatterings, but extreme initial gluon densities, $d N^{g} / d \eta \sim 15,000$, or extreme elastic parton cross sections, $\sim 45 \mathrm{mb}$, are required [18].

In order to verify the existence of a hard scattering and fragmentation component at high $p_{T}$, two-particle

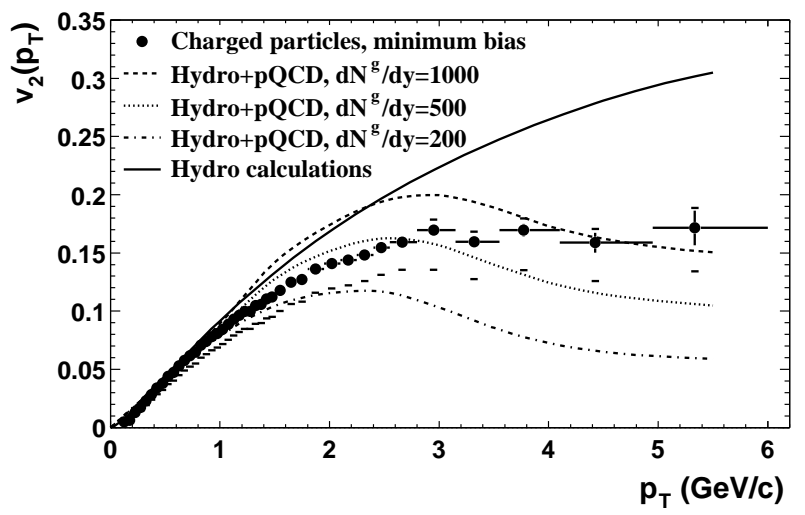

FIG. 3: $v_{2}\left(p_{T}\right)$ for minimum-bias events (circles). The error bars represent the statistical errors and the caps show the systematic uncertainty. The data are compared with hydro+pQCD calculations [9] assuming the initial gluon density $d N^{g} / d y=1000$ (dashed line), 500 (dotted line), and 200 (dashed-dotted line). Also shown are pure hydrodynamical calculations [16] (solid line).

angular correlation measurements are used [13]. In central heavy-ion collisions, it is impossible to reconstruct jets fully due to the large overall particle density. Angular correlations of high $p_{T}$ particles, however, allow for an identification of a hard scattering component on a statistical basis. The fragmentation of high $p_{T}$ partons into several particles results in correlations of hadrons at small $\Delta \eta, \Delta \phi$. In order to isolate this short-range component of the two-particle correlation function, the azimuthal correlations of high $p_{T}$ particles are measured in two regions of relative pseudorapidity. At large $\Delta \eta$, we assume that the azimuthal correlations are free from the fragmentation component.

For the two-particle azimuthal correlation analysis, events containing a trigger particle having $4<p_{T}$ (trig) $<$ $6 \mathrm{GeV} / \mathrm{c}$ and $|\eta|<0.7$ are used. For these events, we measure the relative azimuthal distribution of other charged tracks with $2 \mathrm{GeV} / \mathrm{c}<p_{T}<p_{T}$ (trig) and $|\eta|<0.7$, and the distribution is normalized to the number of high $p_{T}$ trigger particles,

$$
\frac{1}{N_{\text {trigger }}} \frac{d N}{d(\Delta \phi)} \equiv \frac{1}{N_{\text {trigger }}} \frac{1}{\epsilon} \int d \Delta \eta N(\Delta \phi, \Delta \eta) .
$$

$N_{\text {trigger }}$ is the observed number of tracks satisfying the trigger requirement, and $N(\Delta \phi, \Delta \eta)$ is the number of observed pairs as a function of relative azimuth $(\Delta \phi)$ and pseudorapidity $(\Delta \eta)$, and $\epsilon$ is the single track efficiency. Due to the nearly uniform azimuthal acceptance of STAR, no mixed event reference is required. With this definition of the correlation function, the efficiency for finding the trigger particle cancels, and we need only correct the data for the efficiency of finding the lower $p_{T}$ particle. This efficiency is determined using embedding and is $66 \%$ for the tracks used in this analysis. 


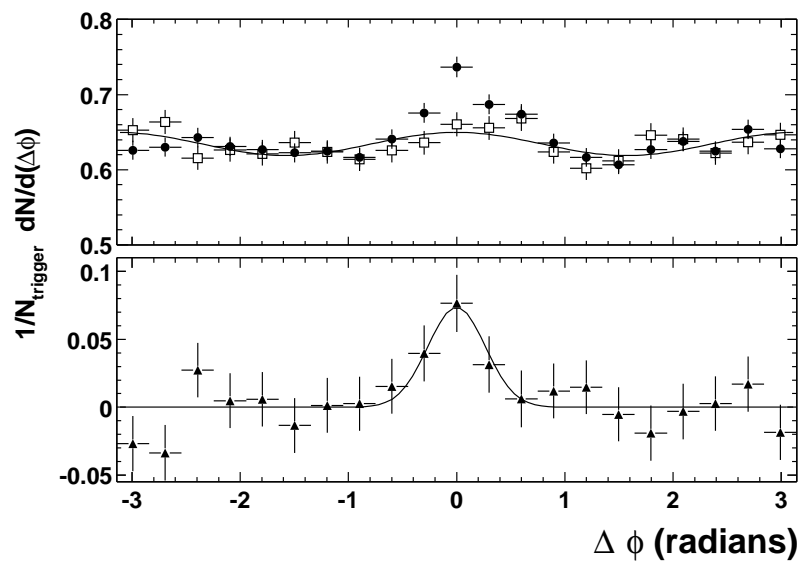

FIG. 4: High $p_{T}$ azimuthal correlation functions for central events. Upper panel: Correlation function for $|\Delta \eta|<0.5$ (solid circles) and scaled correlation function for $0.5<|\Delta \eta|<$ 1.4 (open squares). Lower panel: Difference of the two correlation functions. Also shown are the fits to the data (described in the text).

Figure 4 shows the two-particle azimuthal correlation functions for $|\Delta \eta|<0.5$ and $|\Delta \eta|>0.5$ for central events. Here the small relative pseudorapidity correlation function is absolutely normalized, while the large relative pseudorapidity correlation function has been scaled to match the small $|\Delta \eta|$ correlation function in the region $0.75<|\Delta \phi|<2.25$. There is an enhancement near $\Delta \phi=0$. This short range correlation may be evidence of hard scattering and fragmentation. The large relative pseudorapidity correlation function is fit by a functional form $d N / d(\Delta \phi) \propto 1+2 v_{2}^{2} \cos (2 \Delta \phi)$, expected if the correlations are due entirely to elliptic flow. This fit gives $v_{2}=0.11 \pm 0.02$.

In order to compare this pair-wise method quantitatively to the reaction plane-based $v_{2}$ analysis shown in Figure 1] we construct azimuthal correlation functions with a trigger particle having $2<p_{T}<6 \mathrm{GeV} / \mathrm{c}$. For $|\Delta \eta|>0.5$, the azimuthal correlations give $v_{2}$ results consistent with the reaction plane analysis: $v_{2}=$ $0.203 \pm 0.012,0.160 \pm 0.007$, and $0.091 \pm 0.003$ for the three centrality bins. If no pseudorapidity gap is required between two particles, the $v_{2}$ values are $10-15 \%$ higher.

Also shown in Figure 4 is the difference between the small and large relative pseudorapidity correlation functions. There is an enhancement near zero, and a flat correlation function at larger $\Delta \phi$. A Gaussian fit gives $\sigma=0.27 \pm 0.09$ (stat.) \pm 0.04 (sys.) radians, where the systematic error was estimated by varying the binning and range used to scale the correlation function for $0.5<|\Delta \eta|<1.4$. This width is consistent with earlier observations of jet characteristics in $p p$ collisions at slightly lower 19] and higher 13] energies. The HIJING event generator [20], where hard scattering and fragmentation dominate particle production at these transverse momenta, predicts $\sigma=0.20 \pm 0.01$ for the same kinematic cuts.. Integrating the signal we observe that $4.9 \pm 1.7$ (stat.) \pm 0.4 (sys.) $\%$ of charged particles with $p_{T}$ of $4-6 \mathrm{GeV} / \mathrm{c}$ have an associated charged particle with $p_{T}>2 \mathrm{GeV} / \mathrm{c}$. The systematic error is dominated by the uncertainty in the absolute efficiency determined via the embedding procedure. The contributions of resonance decays and photon conversions were studied and found to be insignificant.

In summary, the measurements of azimuthal anisotropy $v_{2}$ of charged particles with $p_{T}$ of $3-6 \mathrm{GeV} / \mathrm{c}$ reveal a saturation pattern of $v_{2}$ with values that decrease systematically with increasing centrality. This contradicts non-dissipative hydrodynamics which predicts a monotonically increasing $v_{2}$ with increasing $p_{T}$, but the data may be consistent with dissipative dynamics with finite parton energy loss. In addition, a comparison of the twoparticle azimuthal correlation functions for particles with $|\Delta \eta|<0.5$ and $|\Delta \eta|>0.5$ suggests the existence of a short-range correlated component at high $p_{T}$ in addition to underlying global elliptic flow. This may be the first direct evidence at RHIC for hard scattering and parton fragmentation. The data provide important constraints on the theoretical interpretations of the mechanism of high $p_{T}$ particle production in ultra-relativistic heavyion collisions.

We wish to thank the RHIC Operations Group and the RHIC Computing Facility at Brookhaven National Laboratory, and the National Energy Research Scientific Computing Center at Lawrence Berkeley National Laboratory for their support. This work was supported by the Division of Nuclear Physics and the Division of High Energy Physics of the Office of Science of the U.S. Department of Energy, the United States National Science Foundation, the Bundesministerium für Bildung und Forschung of Germany, the Institut National de la Physique Nucléaire et de la Physique des Particules of France, the United Kingdom Engineering and Physical Sciences Research Council, Fundação de Amparo à Pesquisa do Estado de São Paulo, Brazil, the Russian Ministry of Science and Technology and the Ministry of Education of China and the National Science Foundation of China.

[1] STAR Collaboration, K. H. Ackermann et al., Phys. Rev. Lett. 86, 402 (2001).

[2] J.-Y. Ollitrault, Phys. Rev. D 46, 229 (1992).

[3] H. Sorge, Phys. Rev. Lett. 78, 2309 (1997).

[4] M. Gyulassy and M. Plumer, Phys. Lett. B 243, 432 (1990); X.-N. Wang and M. Gyulassy, Phys. Rev. Lett. 68, 1480 (1992).

[5] M. Gyulassy and X.-N. Wang, Nucl. Phys. B 420, 583 (1994); R. Baier, Y. L. Dokshitzer, S. Peigne and D. Schiff, Phys. Lett. B 345, 277 (1995).

[6] PHENIX Collaboration, K. Adcox et al., Phys. Rev. Lett. 88, 022301 (2002).

[7] STAR Collaboration, C. Adler et al., Phys. Rev. Lett. 
89, 202301 (2002).

[8] X.-N. Wang, Phys. Rev. C 63, 054902 (2001).

[9] M. Gyulassy, I. Vitev and X.-N. Wang, Phys. Rev. Lett. 86, 2537 (2001).

[10] S. Voloshin and Y. Zhang, Z. Phys. C 70, 665 (1996); A. M. Poskanzer and S. A. Voloshin, Phys. Rev. C 58, 1671 (1998).

[11] STAR Collaboration, C. Adler et al., Phys. Rev. Lett. 87, 182301 (2001).

[12] M. Anderson et al., Nucl. Instr. Meth. A (2002), RHIC Special Volume, in press.

[13] G. Arnison et al., Phys. Lett. B 118, 173 (1982).

[14] N. Borghini, P. M. Dinh, and J.-Y. Ollitrault, Phys. Rev.
C 64, 054901 (2001).

[15] STAR Collaboration, C. Adler et al., Phys. Rev. C 66, 034904 (2002).

[16] P. Huovinen, P. F. Kolb, U. W. Heinz, P. V. Ruuskanen, and S. A. Voloshin, Phys. Lett. B 503, 58 (2001).

[17] M. Gyulassy, I. Vitev, X.-N. Wang, and P. Huovinen, Phys. Lett. B 526, 301 (2002).

[18] D. Molnar and M. Gyulassy, Nucl. Phys. A 697, 495 (2002).

[19] A. Breakstone et al., Z. Phys. C 23, 1 (1984).

[20] X.-N. Wang, Phys. Rev. D 46, R1900 (1992); Phys. Rev. D 47, 2754 (1993). 